\title{
Invariant Hodge forms and equivariant splittings of algebraic manifolds
}

\author{
by Micha€ Sadowski (Gdańsk)
}

\begin{abstract}
Let $T$ be a complex torus acting holomorphically on a compact algebraic manifold $M$ and let $\mathrm{ev}_{*}: \pi_{1}(T, 1) \rightarrow \pi_{1}\left(M, x_{0}\right)$ be the homomorphism induced by ev : $T \ni t \mapsto t x_{0} \in M$. We show that for each $T$-invariant Hodge form $\Omega$ on $M$ there is a holomorphic fibration $p: M \rightarrow T$ whose fibers are $\Omega$-perpendicular to the orbits. Using this we prove that $M$ is $T$-equivariantly biholomorphic to $T \times M / T$ if and only if there is a subgroup $\Delta$ of $\pi_{1}(M)$ and a Hodge form $\Omega$ on $M$ such that $\pi_{1}(M)=\operatorname{imev} * \times \Delta$ and $\int_{\beta \times \delta} \Omega=0$ for all $\beta \in \mathrm{imev}_{*}$ and $\delta \in \Delta$.
\end{abstract}

Let $T=\mathbb{C}^{n} / B$ be a complex torus acting holomorphically and effectively on a closed complex algebraic manifold $M$. It has been shown in [2] that, if we take an appropriate action of $T$ on $T$, then there is an equivariant fibration $p: M \rightarrow T$ having fibers transversal to the orbits. In particular, a finite covering space $\widehat{M}$ of $M$ is equivariantly biholomorphic to $T \times \widehat{M} / T$. In this paper we consider a more refined variant of this result. Applying the main results of [6] we show that for each $T$-invariant Hodge form $\Omega$ on $M$ there is a $T$-equivariant fibration $p: M \rightarrow T$ whose fibers are $\Omega$-perpendicular to the orbits. We show that the structure group of $p$ depends only on the appropriate periods of $\Omega$. Using this we describe when $M$ is $T$-equivariantly biholomorphic to $T \times M / T$.

Before stating the results of the paper we need some definitions. A smooth fibration $p: M \rightarrow T=\mathbb{C}^{n} / B$ is a t-e fibration if the fibers of $p$ are transversal to the orbits and $x \mapsto p(t x) p(x)^{-1}$ depends on $t \in T$ only (cf. [6, p. 216]). For every $b \in B$ let $b^{\pi}$ denote the corresponding element of $\pi_{1}(T)$. Fix a basis $b_{1}, \ldots, b_{2 n}$ in $B$. Let $\mathrm{ev}_{*}: \pi_{1}(T, 1) \rightarrow \pi_{1}\left(M, x_{0}\right)$ be the homomorphism induced by $T \ni t \mapsto t x_{0} \in M$ and let $\beta_{j}, j=1, \ldots, 2 n$, be the image of $\mathrm{ev}_{*}\left(b_{j}^{\pi}\right)$ in $H_{1}(M, \mathbb{Z})$. Then we have the following.

1991 Mathematics Subject Classification: Primary 32L05; Secondary 55R91, 57S99.

Key words and phrases: holomorphic action, fibration, Hodge form, equivariant splitting, algebraic manifold. 
TheOREM 1. Let $T=\mathbb{C}^{n} / B$ be a complex torus acting holomorphically on a closed algebraic manifold $M$ and let $\Omega$ be a T-invariant Hodge form on $M$. Then there is a holomorphic t-e fibration $p: M \rightarrow T$ whose fibers are $\Omega$-perpendicular to the orbits of the action of $T$. The structure group of $p$ can be reduced to $\mathbb{Z}_{a}^{2 n}$, where $a=\left|\operatorname{det}\left[\int_{\beta_{i} \times \beta_{j}} \Omega\right]\right|$.

Theorem 2. Let $M$ and $T$ be as in Theorem 1. The following conditions are equivalent:

(a) $M$ is $T$-equivariantly biholomorphic to $T \times M / T$,

(b) there is a subgroup $\Delta$ of $\pi_{1}(M)$ and a Hodge form $\Omega$ on $M$ such that

$$
\pi_{1}(M)=\operatorname{imev}_{*} \times \Delta \text { and } \int_{\beta \times \delta} \Omega=0 \text { for all } \beta \in \mathrm{imev}_{*} \text { and } \delta \in \Delta .
$$

The following notation will be used in the proofs of Theorems 1 and 2 . By $\varphi_{u}: M \rightarrow M, u \in \mathbb{C}^{n}$, we shall denote the action of $\mathbb{C}^{n}$ determined by the action of $T=\mathbb{C}^{n} / B$ on $M$. Let $x_{0}$ be a base point of $M$. For every $j=1, \ldots, 2 n, \varphi_{t b_{j}}: M \rightarrow M, t \in[0,1]$, is an $S^{1}$ action on $M$. Let $c_{j}(t)=$ $\varphi_{t b_{j}}\left(x_{0}\right), t \in[0,1]$, and let $X_{j}$ be the vector field on $M$ determined by $t \mapsto \varphi_{t b_{j}}$. It is easy to see that $c_{j}$ belongs to $\beta_{j}$ and $\frac{d c_{j}}{d t}(t)=X_{j}(c(t))$.

We start with the following:

Lemma 1. Let $T=\mathbb{C}^{n} / B, M, \Omega, \beta_{1}, \ldots, \beta_{2 n}$ be as in Theorem $1, \gamma \in$ $\pi_{1}(M), i, j \in\{1, \ldots, 2 n\}$, and $\eta_{j}: T M \ni v \mapsto \Omega\left(v, X_{j}\right) \in \mathbb{C}$. Then
(a) $d \eta_{j}=0$
(b) $\int_{\gamma} \eta_{j} \in \mathbb{Z}$
(c) $\int_{\beta_{i}} \eta_{j}=\int_{\beta_{i} \times \beta_{j}} \Omega=\Omega\left(X_{i}\left(x_{0}\right), X_{j}\left(x_{0}\right)\right)$,
(d) $\operatorname{det}\left[\int_{\beta_{i} \times \beta_{j}} \Omega\right] \neq 0$.

Pr o of. (a) We have

$$
i_{X_{j}} \Omega(v)=\Omega\left(X_{j}, v\right)=-\Omega\left(v, X_{j}\right)=-\eta_{j}(v) .
$$

By the $T$-invariance of $\Omega$,

$$
d i_{X_{j}} \Omega+i_{X_{j}} d \Omega=L_{X_{j}} \Omega=0
$$

so that $d \eta_{j}=-d i_{X_{j}} \Omega=0$.

(b) Let $c:[0,1] \rightarrow M$ be a smooth singular simplex representing the image of $\gamma$ in $H_{1}(M, \mathbb{Z})=\pi_{1}(M) /\left[\pi_{1}(M), \pi_{1}(M)\right]$. The formula $f:[0,1]^{2} \ni$ $(t, s) \mapsto \varphi_{s b_{j}}(c(t)) \in M$ defines a singular cube on $M$. It is easy to see that $f$ is a cycle and

$$
\frac{\partial f}{\partial t}(t, 0)=\frac{d c}{d t}(t), \quad \frac{\partial f}{\partial s}(t, s)=X_{j}(f(t, s)) .
$$


Using this and the $T$-invariance of $\Omega$ we have

$$
\int_{0}^{1} \Omega\left(\frac{\partial f}{\partial t}(t, s), \frac{\partial f}{\partial s}(t, s)\right) d s=\Omega\left(\frac{d c}{d t}(t), X_{j}(c(t))\right),
$$

so that

$$
\begin{aligned}
\int_{c} \eta_{j} & =\int_{0}^{1} \Omega\left(\frac{d c}{d t}(t), X_{j}(c(t))\right) d t \\
& =\int_{0}^{1} \int_{0}^{1} \Omega\left(\frac{\partial f}{\partial t}(t, s), \frac{\partial f}{\partial s}(t, s)\right) d s d t=\int_{f} \Omega \in \mathbb{Z} .
\end{aligned}
$$

(c) By the $T$-invariance of $\Omega, X_{i}$ and $X_{j}$ we have

$$
\begin{aligned}
\int_{\beta_{i}} \eta_{j} & =\int_{c_{i}} \eta_{j}=\int_{0}^{1} \eta_{j}\left(\frac{d c_{i}}{d t}(t)\right) d t=\int_{0}^{1} \eta_{j}\left(X_{i}\left(c_{i}(t)\right) d t\right. \\
& =\int_{0}^{1} \Omega\left(X_{i}\left(c_{i}(t)\right), X_{j}\left(c_{i}(t)\right)\right) d t=\Omega\left(X_{i}\left(x_{0}\right), X_{j}\left(x_{0}\right)\right) .
\end{aligned}
$$

Using arguments similar to those given in the proof of (b) it is easy to verify that

$$
\int_{\beta_{i} \times \beta_{j}} \Omega=\Omega\left(X_{i}\left(x_{0}\right), X_{j}\left(x_{0}\right)\right) .
$$

Thus

$$
\int_{c_{i}} \eta_{j}=\int_{\beta_{i} \times \beta_{j}} \Omega=\Omega\left(X_{i}\left(x_{0}\right), X_{j}\left(x_{0}\right)\right) .
$$

(d) We have $\operatorname{dim}_{\mathbb{C}} T\left(x_{0}\right)=\operatorname{dim}_{\mathbb{C}} T=n$, because every holomorphic, effective action of $T$ on a closed Kähler manifold is almost free (see [2] and Remark 2 below). Let $\Omega_{T}$ denote the restriction of $\Omega$ to $T\left(x_{0}\right)$. Then $\Omega_{T}$ is a Hodge form on $T\left(x_{0}\right)$ so that $\Lambda^{n} \Omega_{T}$ is a volume form on $T\left(x_{0}\right)$. Since $X_{1}\left(x_{0}\right), \ldots, X_{2 n}\left(x_{0}\right)$ is a basis of $T T\left(x_{0}\right)_{x_{0}}$ we have

$$
\operatorname{det}\left[\int_{\beta_{i} \times \beta_{j}} \Omega\right]=\operatorname{det}\left[\Omega\left(X_{i}\left(x_{0}\right), X_{j}\left(x_{0}\right)\right)\right] \neq 0 .
$$

This completes the proof of Lemma 1 .

Let $T O$ be the set of all vectors $v \in T M$ that are tangent to the orbits of the action of $T$. As $T$ acts almost freely, $T O$ is a complex vector bundle. Let $T O^{\perp}=\left\{v \in T M: \forall_{w \in T O} \Omega(v, w)=0\right\}$. Since $\Omega(J v, J w)=\Omega(v, w)$ and $\Omega(J v, w)=-\Omega(v, J w)$ we have

$$
T O^{\perp}=\left\{v \in T M: \forall_{w \in T O} \Omega(v, J w)=0\right\} .
$$


Using this it is easy to see that $T O^{\perp}$ is a complex vector bundle and $T M=$ $T O \oplus T O^{\perp}$.

Let $v \in T M$. Take $v_{0} \in T O$ and $v_{F} \in T O^{\perp}$ such that $v=v_{0}+v_{F}$. Let $E(v)$ be the invariant vector field on $T$ such that $v_{0}$ is tangent to the orbit of the one-parameter subgroup generated by $E(v)$. For every $u \in \mathbb{C}^{n}$ let $I^{-1}(u) \in L(T)$ be the invariant vector field on $T$ such that $u$ is tangent to the one-parameter subgroup generated by $I^{-1}(u)$. Consider the 1 -form

$$
\omega: T M \ni v \mapsto(I \circ E)(v) \in \mathbb{C}^{n} .
$$

Applying Lemma 1 we show the following:

Lemma 2. Let $M, T, B, \Omega, X_{1}, \ldots, X_{2 n}, \beta_{1}, \ldots, \beta_{2 n}$ be as above, $\gamma \in$ $\pi_{1}(M), j \in\{1, \ldots, 2 n\}, a_{i j}=\Omega\left(X_{i}, X_{j}\right),\left[b_{i j}\right]=\left[a_{i j}\right]^{-1}$, and let $a=$ $\left|\operatorname{det}\left[a_{i j}\right]\right|$. Then

(a) $\omega=\sum_{i=1}^{2 n} \omega_{i} b_{i}$, where $\omega_{i}(v)=\sum_{j=1}^{2 n} b_{j i} \Omega\left(v, X_{j}\right)$,

(b) $\omega$ is a holomorphic 1-form,

(c) $\int_{\gamma} a \omega \in B$,

(d) $\int_{\beta_{j}} \omega=b_{j}$.

Proof. (a) Take $x \in M, v \in T M_{x}$ and $c_{1}, \ldots, c_{2 n} \in \mathbb{C}$ such that $v_{0}=\sum_{j=1}^{2 n} c_{j} X_{j}(x)$. Then

$$
\Omega\left(v, X_{i}\right)=\Omega\left(v_{0}, X_{i}\right)=\sum_{j=1}^{2 n} c_{j} \Omega\left(X_{j}, X_{i}\right)=\sum_{j=1}^{2 n} c_{j} a_{j i}
$$

so that

$$
c_{i}=\sum_{j=1}^{2 n} b_{j i} \Omega\left(v, X_{j}\right)=\omega_{i}(v) .
$$

Since $(I \circ E)\left(X_{i}(x)\right)=b_{i}$ we have

$$
\omega(v)=\sum_{i=1}^{2 n} c_{i} b_{i}=\sum_{i=1}^{2 n} \omega_{i}(v) b_{i} .
$$

(b) By (a) and by Lemma 1,

$$
d \omega=\sum_{i=1}^{2 n} d \omega_{i} b_{i}=\sum_{i=1}^{2 n} \sum_{j=1}^{2 n} b_{j i} d \eta_{j} b_{i}=0 .
$$

It is easy to see that $\omega \circ J=i \omega$. As $\omega$ is closed this implies that $\omega$ is holomorphic.

(c) By Lemma 1(b), $\int_{\gamma} \eta_{i} \in \mathbb{Z}$. Since

$$
a b_{i j}=\left|\operatorname{det}\left[a_{p q}\right]\right| b_{i j} \in \mathbb{Z}
$$


we have

$$
\int_{\gamma} a \omega_{i}=\sum_{j=1}^{2 n} a b_{j i} \int_{\gamma} \eta_{j} \in \mathbb{Z}
$$

so that

$$
\int_{\gamma} a \omega=\sum_{i=1}^{2 n} \int_{\gamma} a \omega_{i} b_{i} \in B
$$

(d) By the definition of $\omega, \omega\left(X_{j}\left(c_{j}(t)\right)\right)=b_{j}$. As $c_{j}:[0,1] \ni t \mapsto \varphi_{t b_{j}}\left(x_{0}\right)$ belongs to $\beta_{j}$,

$$
\int_{\beta_{j}} \omega=\int_{0}^{1} \omega\left(\frac{d c_{j}}{d t}(t)\right) d t=\int_{0}^{1} \omega\left(X_{j}\left(c_{j}(t)\right)\right) d t=\int_{0}^{1} b_{j} d t=b_{j} .
$$

Proof of Theorem 1. By Lemma 2 and by [6, Lemma 1$]$ the formula $p(x)=\int_{x_{0}}^{x} a \omega \bmod B$ defines a well defined holomorphic map $p: M \rightarrow T$. Note that

$$
\left(p_{*} \circ \mathrm{ev}_{*}\right)\left(b_{j}^{\pi}\right)=\left(\int_{\beta_{j}} a \omega\right)^{\pi}=a b_{j}^{\pi}
$$

(compare [5, Lemma 1.2]). It is easy to see that $\omega$ is $T$-invariant. By $[6, \S 1]$, $p$ is a holomorphic t-e fibration.

Let $\mathcal{F}(p)$ be the foliation of $M$ whose leaves are connected components of the fibers of $p$, let $L$ be a leaf of $\mathcal{F}(p)$ containing the base point $x_{0}$, let $x \in L$, and let $v \in T L_{x}$. Take a smooth path $c:[0,1] \rightarrow M$ joining $x_{0}$ to $x$ such that $c^{\prime}(1)=v$. Then

$$
\int_{0}^{t} \omega\left(c^{\prime}(\tau)\right) d \tau=\int_{x_{0}}^{c(t)} \omega=p(c(t))=0 \bmod B
$$

so that $\omega\left(c^{\prime}(t)\right)=0$ for $t \in[0,1]$. In particular, $E(v)=0$ and $v \in T O^{\perp}$. As $p$ is a t-e fibration each element of $T$ carries the leaves of $p$ onto the leaves of $p$. Using this it is easy to see that the fibers of $p$ are $\Omega$-perpendicular to the orbits. Since $\pi_{1}(T) / \mathrm{im}(p \circ \mathrm{ev})_{*} \cong \mathbb{Z}_{a}^{2 n}$, the structure group of $p$ can be reduced to $\mathbb{Z}_{a}^{2 n}$ (see [5, Proposition 2.1]).

Proof of Theorem 2. (a) $\Rightarrow$ (b). Fix a biholomorphic equivariant map $M \rightarrow T \times F$. Let $\Omega_{T}$ and $\Omega_{F}$ be $(1,1)$-forms on $M$ induced by some Hodge forms on $T$ and $F$ respectively. Then $\Omega=\Omega_{T}+\Omega_{F}$ is a Hodge form on $M$. It is clear that $\Omega(v, w)=0$ for $v \in T T_{x}, w \in T F_{x}, x \in M$. Let $\beta_{i} \in H_{1}(T, \mathbb{Z}), i \in\{1, \ldots, 2 n\}$, be as in Theorem 1 , and let $\delta \in H_{1}(F, \mathbb{Z})$. Arguments similar to those given in the proof of Lemma 1(b) show that $\int_{\beta_{i} \times \delta} \Omega=0$. 
(b) $\Rightarrow$ (a). Averaging $\Omega$ we can assume that $\Omega$ is $T$-invariant. Let $\omega$ and $\omega_{i}$ be as in the proof of Theorem 1 and let $\Delta_{H}$ be the image of $\Delta$ in $H_{1}(M, \mathbb{Z})$. If $u \in H_{1}(M, \mathbb{Z})$ then $u=\sum_{j=1}^{2 n} k_{j} \beta_{j}+u_{F}$ for some $k_{1}, \ldots, k_{2 n} \in \mathbb{Z}$ and $u_{F} \in \Delta_{H}$. Clearly $\int_{u_{F}} \omega_{i}=0$ so that

$$
\int_{u} \omega_{i}=\sum_{j=1}^{2 n} k_{j} \int_{\beta_{j}} \omega_{i} \in \mathbb{Z}
$$

and accordingly $\int_{u} \omega \in B$. The arguments given in the proof of Theorem 1 show that

$$
q: M \ni x \mapsto \int_{x_{0}}^{x} \omega \bmod B \in \mathbb{C}^{n} / B=T
$$

is a well defined holomorphic t-e fibration. By Lemma $2,(q \circ \mathrm{ev})_{*}\left(b_{i}^{\pi}\right)=$ $\left(\int_{\beta_{i}} \omega\right)^{\pi}=b_{i}^{\pi}$ (cf. [5, Lemma 1.2]). Hence $q_{*} \circ \mathrm{ev}_{*}$ is an epimorphism and $q$ is a trivial fibration. This completes the proof of Theorem 2.

R e mark 1. (a) Natural examples of holomorphic toral actions on algebraic manifolds and the arising equivariant splittings were discussed in $[1,3]$.

(b) The theorem of complete reducibility of Poincaré (see e.g. [4, §19, Theorem 1]) is a particular case of Theorem 1 . To see this let $T_{0}$ be an abelian variety and let $T=\mathbb{C}^{n} / B$ be a complex torus contained in $T_{0}$. Then $T$ acts holomorphically (and freely) on $T_{0}$. By Theorem 1 there is a holomorphic t-e fibration $p: T_{0} \rightarrow T$ associated with a $T_{0}$-invariant Hodge form $\Omega$ on $T_{0}$. Any connected component $L$ of a fiber of $p$ is a leaf of a $T$-invariant foliation $F(p)$. Since the leaves of $F(p)$ are covered by complex hyperplanes, $L$ is a complex torus. It is easy to see that $\varepsilon: T \times L \ni(t, u) \mapsto t u \in T_{0}$ is an epimorphism with a finite kernel.

Remark 2. Using the arguments similar to those given in the proof of Lemma 1 it is possible to give a simple proof of the almost freeness of an effective holomorphic action of a complex torus on a closed Kähler manifold $M$. It goes as follows. Let $T=\mathbb{C}^{n} / B$ be a complex torus acting on $M$, let $b \in B$, and let $\varphi_{t b}: M \rightarrow M, t \in[0,1]$, be an $S^{1}$ action embedded in the action of $T$. Let $X$ be the vector field on $M$ determined by the $S^{1}$ action, let $c_{b}$ be any nontrivial orbit of the action of $\varphi_{t b}, t \in[0,1]$, and let $\Omega$ be any invariant Kähler form on $M$. The formula $\eta(v)=-\frac{1}{i} \Omega(v, J X)=$ $\frac{1}{i} \Omega(J v, X)$ defines a closed (see Lemma 1 ) 1 -form on $M$. For every $v \neq 0$ we have $\frac{1}{i} \Omega(J v, v)>0$ so that

$$
\int_{c_{b}} \eta=\frac{1}{i} \int_{0}^{1} \Omega(J X(c(t)), X(c(t))) d t>0 .
$$

In particular, the isotropy group of every orbit of the $S^{1}$ action is discrete. 


\section{References}

[1] E. Calabi, On Kähler manifolds with vanishing canonical class, in: Algebraic Geometry and Topology, Princeton Univ. Press, 1957, 78-89.

[2] J. B. Carrell, Holomorphically injective complex toral actions, in: Proc. Second Conference on Compact Transformation Groups, Part 2, Lecture Notes in Math. 299, Springer, 1972, 205-236.

[3] J. Matsushima, Holomorphic vector fields and the first Chern class of a Hodge manifold, J. Differential Geom. 3 (1969), 477-480.

[4] D. Mumford, Abelian Varieties, Oxford Univ. Press, Oxford, 1970.

[5] M. Sadowski, Equivariant splittings associated with smooth toral actions, in: Algebraic Topology, Proc., Poznań 1989, Lecture Notes in Math. 1474, Springer, 1991, 183-193.

[6] - Holomorphic splittings associated with holomorphic complex torus actions, Indag. Math. (N.S.) 5 (1994), 215-219.

Department of Mathematics

Gdańsk University

Wita Stwosza 57

80-952 Gdańsk, Poland

E-mail: matms@paula.univ.gda.pl 